International Journal of Pure and Applied Mathematics

Volume 107 No. 2 2016, 289-300

ISSN: 1311-8080 (printed version); ISSN: 1314-3395 (on-line version)

url: http://www.ijpam.eu

doi: 10.12732/ijpam.v107i2.1

\title{
NUMERICAL SIMULATION USING THE HOMOTOPY PERTURBATION METHOD FOR A THIN LIQUID FILM OVER AN UNSTEADY STRETCHING SHEET
}

\author{
Majeed A. Yousif ${ }^{1} \S$, Bewar A. Mahmood ${ }^{2}$ \\ Karmina K. Ali ${ }^{3}$, Hajar F. Ismael ${ }^{4}$ \\ ${ }_{1,3,4}$ University of Zakho \\ Kurdistan Region, IRAQ \\ ${ }^{2}$ University of Duhok \\ Kurdistan Region, IRAQ
}

\begin{abstract}
In this article, the flow of liquid film over an unsteady elastic stretching surface is analyzed. Similarity transformations are used to transform the governing equations to a nonlinear ordinary differential equation. The differential equation reformulated to system of Voltera integral equations and solved analytically using the new technique of numerical solution called homotopy perturbation method. The results of the proposed method are compared with previously published work and the results are found to be in an excellent agreement. Also, discussed and presented graphically the effects of various parameters Darcy number and unsteadiness parameter.
\end{abstract}

AMS Subject Classification: $74 \mathrm{~K} 35,65-\mathrm{XX}$

Key Words: homotopy perturbation method, thin liquid film, Voltera integral equations, stretching sheet

Received: February 9, 2016

Published: April 3, 2016

${ }^{\S}$ Correspondence author (c) 2016 Academic Publications, Ltd. url: www.acadpubl.eu 


\section{Introduction}

In recent years the flow in a thin liquid film over an unsteady stretching surface got more attention because of their application in many field of science and technology such as fiber coating, food processing, transpiration cooling and others. Crane was the first among others researchers to consider the steady two-dimensional flow of a Newtonian fluid over an stretching flat sheet which moves in its own plane with a velocity varying linearly with the distance from a fixed point [7]. Wang have studied the three dimensional flow that due to a stretching surface and to a stretching surface in a rotating fluid [24], Wang was the first researcher who investigated the flow in thin liquid films driven by an unsteady stretching sheet [25]. Usha and Sridharan assumed a similar problem of axisymmetric flow in a thin liquid film. This problem developed by some authors, after adding heat transfer in a thin liquid over an unsteady stretching sheet [22]. Anderson et. al. expanded the problem of Wang and studied liquid films on an unsteady stretching surface under the effect of heat transform and used new exact similarity solution [6]. Wang and Pop analyzed flow of a powerlaw fluid film by the unsteady stretching of a horizontal elastic surface by using homotopy analysis method [23]. Also, Abbas et. al. solved developed nonlinear equation of a thin liquid film of second grade fluid over an unsteady stretching surface analytically by using the homotopy analysis method [1].

Abel et. al. studied boundary layer behavior in a liquid film driven by an unsteady stretching sheet including the effects of variable transverse magnetic field and concluded that the effect of transverse magnetic field on a viscous incompressible conducting fluid is suppress the velocity field which in turn causes the enhancement of the temperature field [3]. Noor and Hashim considered flow in the effects of thermocapillarity and a magnetic and heat transfer in a liquid film over an unsteady elastic stretching surface and the differential equations are solved numerically by using the homotopy analysis method [20]. Laraqi et al. analytically studied the thermo-hydrodynamic behaviour of a thin lubricant film [16]. Makinde and Aziz presented boundary layer flow of a nanofluid past a stretching sheet with a convective boundary condition numerically using fourth-fifth order Runge-Kutta-Fehlberg method [17]. Khader and Megahed solved numerically the flow and heat transfer in a thin liquid film over an unsteady stretching sheet in a saturated porous medium in the presence of thermal radiation by using the finite-difference method [15]. Recently Mairy investigated the flow and heat transfer of a thin liquid film over an unsteady porous stretching sheet in the presence of suction or injection by considering a full set of momentum equations [21]. In recent years, the application of the 
homotopy perturbation method [13] in nonlinear problems has been developed by scientists and engineers, because this method continuously deforms the difficult problem under study into a simple problem which is easy to solve. The homotopy perturbation method [11], proposed first by He in 1998 and was further developed and improved by He (see $[12,13,14]$ ). Recently, the applications of homotopy perturbation have appeared in the works of many scientists $[8,10,5,18,9]$.In this work we will transfer governing equations to a nonlinear ordinary differential equation and to a system of Voltera integral equations to find the approximate solution for a thin liquid film over an unsteady stretching sheet by homotopy perturbation method (HPM) and we will compare the results by HPM with obtained by homotopy analysis method (HAM) (see [20]).

\section{Mathematical Model}

Unsteady boundary layer of an incompressible flow in two dimensional see figure (1), derived by a stretching surface with velocity $U=\frac{b x}{1-a t}$, where both $a, b$ are positive and have dimension $t^{-1}$.

The governing Navier-Stokes, and continuity equation in two dimension that covers the problem is given by:

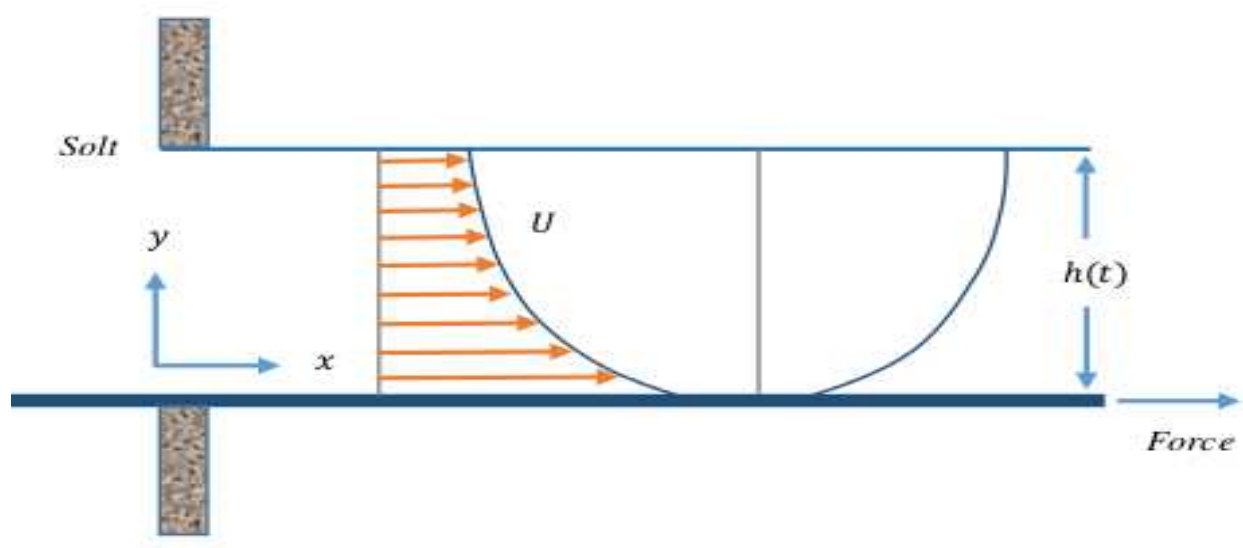

Figure 1: Schematic of the physical system.

$$
\frac{\partial u}{\partial x}+\frac{\partial v}{\partial y}=0
$$




$$
\begin{gathered}
\frac{\partial u}{\partial t}+u \frac{\partial u}{\partial x}+v \frac{\partial u}{\partial y}=\frac{\mu}{\rho} \frac{\partial^{2} u}{\partial y^{2}}-\frac{\mu}{\rho k} u \\
u=U, \quad v=0 \quad \text { at } \quad y=0, \\
\frac{\partial u}{\partial y}=0, \quad v=\frac{d h}{d t} \quad \text { at } \quad y=h,
\end{gathered}
$$

where $U$ is the surface velocity of the stretching sheet and the flow is caused by stretching the elastic surface at $y=0$. One can be notice that at $t=0$ (initial motion), equations (1) and (2) describe the steady flow over a stretching surface.

We introduce the following dimensionless transformations $f$ and $\theta$ and similarity variable $\eta$.

$$
\begin{gathered}
\eta=\sqrt{\frac{b}{v}}(1-a t)^{-\frac{1}{2}} \beta^{-1} y \\
u=b x(1-a t)^{-1} f(\eta) \\
v=-\sqrt{\frac{b v}{1-a t}} \beta f(\eta) \\
\beta=\sqrt{\frac{b}{v(1-a t)}} h(t)
\end{gathered}
$$

using equations (5) and (8)into equations (1) and (4), the mathematical model of problem will be transform into the following nonlinear ordinary differential equation.

$$
f^{\prime \prime \prime}+\gamma\left[f f^{\prime \prime}-\frac{S}{2} \eta f^{\prime \prime}-f^{\prime 2}-(S+D) f^{\prime}\right]=0
$$

subject to the boundary conditions:

$$
\begin{gathered}
f(0)=0, \quad f^{\prime}(0)=1 \\
f^{\prime \prime}(1)=0,
\end{gathered}
$$

where primes denote differentiation with respect to $\eta, S=\frac{a}{b}$ is the unsteadiness parameter, $\gamma$ is the dimensionless film thickness and $D=\frac{\mu(1-a t}{\rho b k}$ is the Darcy number. 


\section{Basic Idea of Homotopy Perturbation Method using Volterra Integral Equations}

Aslam Noor and Mohyud-Din defined homotopy perturbation method after transforming to the system of Volterra integral equations (see $[4,11]$ ).

$$
F(t)=G(t)+\gamma \int_{0}^{t} K(t, s) F(s) d s
$$

where: $F(t)=\left(f_{1}(t), f_{2}(t), \cdots, f_{n}(t)\right)^{T}$,

$G(t)=\left(g_{1}(t), g_{2}(t), \cdots, g_{n}(t)\right)^{T}$,

$K(t, s)=\left[K_{i j}(t, s)\right], \quad i=1,2, \cdots, n ; \quad j=1,2, \cdots, n$.

to explain the homotopy perturbation method. We consider a general equation of the type,

$$
L(u)=0
$$

where $L$ is any integral or differential operator. We define a convex homotopy $H(u, p)$ by

$$
H(u, p)=(1-p) F(u)+p L(u),
$$

where $F(u)$ is a functional operator with known solutions $v_{0}$, which can be obtained easily. It is clear that, for

$$
H(u, p)=0
$$

we have

$$
\begin{aligned}
& H(u, 0)=F(u), \\
& H(u, 1)=L(u) .
\end{aligned}
$$

This shows that $H(u, p)$ continuously traces an implicitly defined curve from a starting point $H\left(v_{0}, 0\right)$ to a solution function $H(f, 1)$. The embedding parameter monotonically increases from zero to unit as the trivial problem $F(u)=0$ continuously deforms the original problem $L(u)=0$. The embedding parameter $p \in(0,1]$ can be considered as an expanding parameter see $[11,12,14,19,2]$. The homotopy perturbation method uses the homotopy parameter $p$ as an expanding parameter to obtain

$$
u=\sum_{i=0}^{\infty} p^{i} u_{i}=u_{0}+p u_{1}+p^{2} u_{2}+p^{3} u_{3}+\cdots
$$


If $p \rightarrow 1$, then(18) corresponds to (14) and becomes the approximate solution of the form

$$
f=\lim _{p \rightarrow 1} u=\sum_{i=0}^{\infty} u_{i} .
$$

It is well known that series (19) is convergent for most of the cases and also the rate of convergence depends on $L(u)$ see[11]. We assume that (19) has a unique solution. Consider the $i^{\text {th }}$ equation of (12), we take

$$
\left\{\begin{array}{l}
f_{1}(t)=\sum_{i=0}^{\infty} p^{i} u_{i} \\
f_{2}(t)=\sum_{i=0}^{\infty} p^{i} v_{i} \\
f_{3}(t)=\sum_{i=0}^{\infty} p^{i} s_{i} \\
\vdots
\end{array}\right.
$$

The comparisons of like powers of $p$ give solutions of various orders [4] and [11].

\section{Analytical Approximations by Means of the Homotopy Perturbation Method}

Our aim in this paper is to solve the problem (9) with the boundary conditions $(10,11)$ using the homotopy perturbation method. Now may be reformulated as a system of integral equations and apply homotopy perturbation method.

Using the defined transformation $\frac{d f}{d \eta}=q(\eta), \frac{d q}{d \eta}=k(\eta)$, we rewrite the equation(9) as a system of differential equations

$$
\left\{\begin{array}{l}
\frac{d f}{d \eta}=q(\eta) \\
\frac{d q}{d \eta}=k(\eta) \\
\frac{d k}{d \eta}=-\gamma\left[f(\eta) k(\eta)-\frac{S}{2} \eta k(\eta)-q(\eta)^{2}-(S+D) q(\eta)\right]
\end{array}\right.
$$

with $f_{0}(\eta)=0, \quad q_{0}(\eta)=1, \quad k_{0}(\eta)=A$.

The above system of differential equation can be written as a system of 
integral equations

$$
\left\{\begin{array}{l}
f(\eta)=0+\int_{0}^{\eta} q(\eta) d \eta \\
q(\eta)=1+\int_{0}^{\eta} k(\eta) d \eta \\
k(\eta)=A+\int_{0}^{\eta}-\gamma\left[f(\eta) k(\eta)-\frac{S}{2} \eta k(\eta)-q(\eta)^{2}-(S+D) q(\eta)\right] d \eta
\end{array}\right.
$$

Applying the convex homotopy method, we have

$$
\left\{\begin{array}{l}
f_{0}+p f_{1}+p^{2} f_{2}+p^{3} f_{3}+\cdots=0+p \int_{0}^{\eta}\left(q_{0}+p q_{1}+p^{2} q_{2}+\cdots\right) d \eta \\
q_{0}+p q_{1}+p^{2} q_{2}+p^{3} q_{3}+\cdots=1+p \int_{0}^{\eta}\left(k_{0}+p k_{1}+p^{2} k_{2}+\cdots\right) d \eta \\
k_{0}+p k_{1}+p^{2} k_{2}+p^{3} k_{3}+\cdots=A+p \int_{0}^{\eta}\left\{-\gamma\left[\left(f_{0}+p f_{1}+p^{2} f_{2}+\cdots\right)\right.\right. \\
\quad\left(k_{0}+p k_{1}+p^{2} k_{2}+\cdots\right) \\
\quad-\frac{S}{2} \eta\left(k_{0}+p k_{1}+p^{2} k_{2}+\cdots\right)-\left(q_{0}+p q_{1}+p^{2} q_{2}+\cdots\right)^{2} \\
\left.\left.\quad-(S+D)\left(q_{0}+p q_{1}+p^{2} q_{2}+\cdots\right)\right] d \eta\right\}
\end{array}\right.
$$

Comparing the coefficient of like powers of $p$

$$
\begin{gathered}
p^{0}:\left\{\begin{array}{l}
f_{0}=0, \\
q_{0}=1, \\
k_{0}=A .
\end{array}\right. \\
p^{1}:\left\{\begin{array}{l}
f_{1}=\eta, \\
q_{1}=A \eta, \\
k_{1}=\gamma\left(\frac{1}{4} A \eta^{2} S+D \eta+\eta+\eta S\right) .
\end{array}\right. \\
p^{2}:\left\{\begin{array}{l}
f_{2}=\frac{A \eta^{2}}{2}, \\
q_{2}=\frac{1}{4} \gamma\left(\frac{1}{3} A \eta^{3} S+2 \eta^{2}(D+S+1)\right), \\
k_{2}=\frac{1}{8} \gamma\left(4 A \eta^{2}(D+S)+\frac{1}{4} A \eta^{4} \gamma S^{2}+\frac{4}{3} \eta^{3} \gamma S(D+S+1)\right) .
\end{array}\right.
\end{gathered}
$$




$$
\begin{gathered}
p^{3}:\left\{\begin{array}{l}
f_{3}=\frac{1}{12} \gamma\left(\frac{1}{4} A \eta^{4} S+2 \eta^{3}(D+S+1)\right) \\
q_{3}=\frac{1}{96} \gamma\left(16 A \eta^{3}(D+S)+\frac{3}{5} A \eta^{5} \gamma S^{2}+4 \eta^{4} \gamma S(D+S+1)\right) \\
k_{3}=\frac{1}{1920} \eta^{3} \gamma\left(640 A^{2}+5 A \eta \gamma S\left(32 D+\eta^{2} \gamma S^{2}+32 S-40\right)\right. \\
\quad+32 \gamma(D+S+1)\left(10 D+\eta^{2} \gamma S^{2}+10 S-40\right) .
\end{array}\right. \\
p^{4}:\left\{\begin{array}{r}
f_{4}=\frac{1}{96} \gamma\left(4 A \eta^{4}(D+S)+\frac{1}{10} A \eta^{6} \gamma S^{2}+\frac{4}{5} \eta^{5} \gamma S(D+S+1)\right), \\
q_{4}=\frac{1}{40320} \eta^{4} \gamma\left(3360 A^{2}+3 A \eta \gamma S\left(224 D+5 \eta^{2} \gamma S^{2}+224 S-280\right)\right. \\
+112 \gamma(D+S+1)\left(15 D+\eta^{2} \gamma S^{2}+15 S-60\right) \\
k_{4}= \\
\quad \frac{1}{3840} \gamma^{2}\left(16 \eta^{5} S\left(9 A^{2}+2 \gamma\left(3 D^{2}+D(6 S-11)+3 S^{2}-11 S-14\right)\right)\right. \\
+160 A \eta^{4}\left(D^{2}+D(2 S-3)+S^{2}-3 S+2\right)+\frac{92}{3} A \eta^{6} \gamma S^{2}(D+S-2) \\
+\frac{5}{8} A \eta^{8} \gamma^{2} S^{4}+\frac{32}{7} \eta^{7} \gamma^{2} S^{3}(D+S+1) .
\end{array}\right.
\end{gathered}
$$

The solution is given as

$$
\begin{aligned}
f(\eta)= & \eta+\frac{A \eta^{2}}{2}+\frac{1}{12} \gamma\left(\frac{1}{4} A \eta^{4} S+2 \eta^{3}(D+S+1)\right) \\
& +\frac{1}{96} \gamma\left(4 A \eta^{4}(D+S)+\frac{1}{10} A \eta^{6} \gamma S^{2}+\frac{4}{5} \eta^{5} \gamma S(D+S+1)\right)+O\left(\eta^{6}\right)
\end{aligned}
$$

Imposing the boundary condition at $\eta=1$ leads to

$$
A=-\frac{16\left(6 D \gamma+D \gamma^{2} S+6 \gamma+\gamma^{2} S^{2}+\gamma^{2} S+6 \gamma S\right)}{3\left(16 D \gamma+\gamma^{2} S^{2}+24 \gamma S+32\right)},
$$

The series solution at the $4^{\text {th }}$-order approximation is given as

$$
\begin{aligned}
& f(\eta)=\eta-\frac{8 \eta^{2} \gamma(D+S+1)(\gamma S+6)}{48 D \gamma+3 \gamma^{2} S^{2}+72 \gamma S+96}+\frac{1}{12} \gamma\left(-\frac{16 \eta^{4} \gamma S(D+S+1)(\gamma S+6)}{48 D \gamma+3 \gamma^{2} S^{2}+72 \gamma S+96}\right) \\
& +\frac{1}{12} \gamma\left(2 \eta^{3}(D+S+1)+\frac{1}{4}\right) \\
& \quad+\frac{1}{96} \gamma\left(-\frac{16 \eta^{6} \gamma^{2} S^{2}(D+S+1)(\gamma S+6)}{48 D \gamma+3 \gamma^{2} S^{2}+72 \gamma S+96}-\frac{16 \eta^{4} \gamma(D+S)(D+S+1)(\gamma S+6)}{48 D \gamma+3 \gamma^{2} S^{2}+72 \gamma S+96}\right) \\
& \quad+\frac{1}{96} \gamma\left(\frac{4}{5} \eta^{5} \gamma S(D+S+1)+\frac{41}{10}\right)+O\left(\eta^{6}\right) .
\end{aligned}
$$


Table 1: Comparison for values of $f^{\prime \prime}(0)$ and $\gamma$.

\begin{tabular}{cccc}
\hline \hline & & Present work & Noor and Hashim $([20])$ \\
\hline \hline$S$ & $\gamma$ & $f^{\prime \prime}(0)$ & $f^{\prime \prime}(0)$ \\
\hline 1.4 & 0.674089 & -1.07873 & -1.01278 \\
1.6 & 0.331976 & -0.667678 & -0.64240 \\
1.8 & 0.127013 & -0.314711 & -0.309138 \\
\hline \hline
\end{tabular}

Table 2: Comparison for values of $\gamma$ and $f^{\prime \prime}(0)$ using $4^{\text {th }}$-order HPM approximation for several values of $D$ and $S$.

\begin{tabular}{c|ccc||c|ccc}
\hline \hline & $D$ & $\gamma$ & $f^{\prime \prime}(0)$ & & $S$ & $\gamma$ & $f^{\prime \prime}(0)$ \\
\hline \multirow{4}{*}{$S=0.4$} & 0.0 & 5.56880 & -3.78339 & & 0.0 & 5.56882 & -3.49185 \\
& 0.2 & 5.11978 & -3.45634 & & 0.1 & 5.11977 & -3.32718 \\
& 0.4 & 4.43018 & -3.11766 & $D=0.5$ & 0.3 & 4.43016 & -3.08295 \\
& 0.6 & 4.05661 & -2.93135 & & 0.5 & 4.05660 & -2.96290 \\
& 0.8 & 3.96461 & -2.86144 & & 0.7 & 3.96459 & -2.95749 \\
& 1.0 & 3.36321 & -2.63709 & & 0.9 & 3.36320 & -2.76164 \\
\hline \hline
\end{tabular}

\section{Results and Discussion}

A comparison with the present work with the homotopy analysis method [20] is agree very well as we can see from Table 1, So, the variations of the dimensionless film thickness $\gamma$, skin-friction coefficient in terms of $f^{\prime \prime}(0)$ for the parameters governing the flow. From Table 2, we can observe that, when we decrease the thickness of the thin film are increasing both of the Darcy number and the unsteadiness parameter.

Fig.2,(a) show the effect of the Darcy parameter $D$ on the velocity profile. It is clear that when Darcy parameter increases along the sheet and the reverse is true away from the sheet causes the velocity will decrease.

Fig.2,(b) demonstrates the effect of the unsteadiness parameter $S$ on the velocity profile. The results show that when an increase in the unsteadiness parameter $S$, the velocity increases along the surface. 


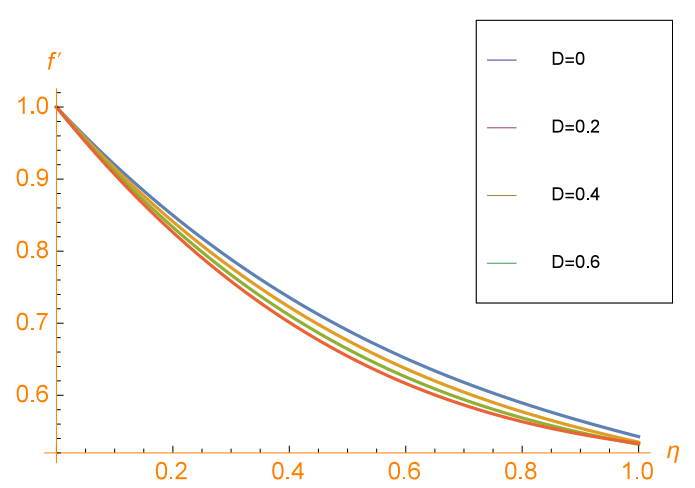

(a) Various values of $D$ with $S=0.8$

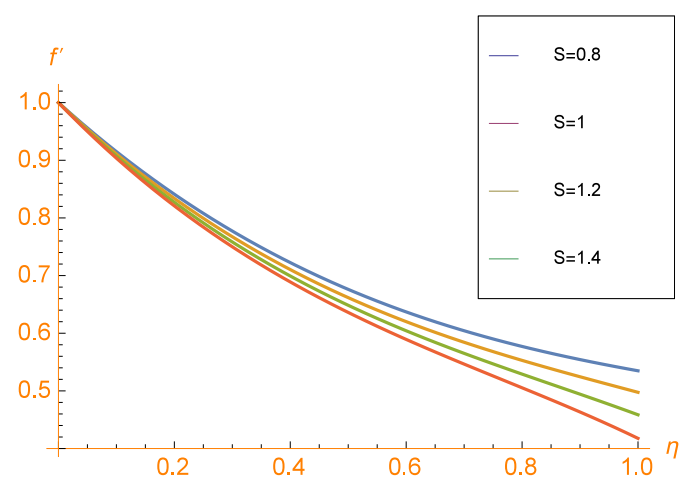

(b) Various values of $S$ with $D=0.2$

Figure 2: The behavior of velocity distribution

\section{Conclusion}

In this paper, the homotopy perturbation method has been successfully applied to find the approximate solution for a thin liquid film over an unsteady stretching sheet. The nonlinear ordinary differential equation considered as transformed governing equation and reformulated to system of Voltera integral equations and solved numerically using homotopy perturbation method (HPM) and compared with homotopy analysis method (HAM), it may be conclude that HPM is powerful mathematical tool for solving such problems. Also, we show that the results of present work has a good agreement with HAM. We studied the effective of various parameters Darcy number and unsteadiness parameter with results and graphically. All results and graphs obtained by using 


\section{Mathematica version 10.}

\section{References}

[1] Abbasa Z, Hayata T, Sajidb M, Asgharc S, Unsteady flow of a second grade fluid film over an unsteadystretching sheet, Mathematical and Computer Modelling, 48 (2008), 518-526.

[2] Abbasbandy S., A numerical solution of Blasius equation by Adomians decomposition method and comparison with homotopy perturbation method, Chaos Solitons Fractals, 31 (2007), 257-260.

[3] Abel M.S, Mahesha N, Tawade J, Heat transfer in a liquid film over an unsteady stretching surface with viscous dissipation in presence of external magnetic field, J. Applied Mathematical Modelling, 33 (2009), 3430-3441.

[4] Aslam Noor M., Mohyud-Din S.T., Homotopy perturbation method for solving sixthorder boundary value problems, Computers and Mathematics with Applications, 55 (2008), 2953-2972.

[5] Ali Demir, Sertac Erman, Berrak Özgür, Esra Korkmaz, Analysis of the new homotopy perturbation method for linear and nonlinear problems, Boundary Value Problems. 61 (2013).

[6] Anderson H.I, Aarseth J.B, Dandapat B.S, Heat transfer in a liquid film on an unsteady stretching surface, Int. J. Heat Mass Transfer, 43 (2000), 69-74.

[7] Crane L.J, flow past a stretching plate, Zeitschrift für angewandte Mathematik und Physik, 4 (1970), 645-647.

[8] El-Shahed M, Application of Hes homotopy perturbation method to Volterras integrodifferential equation, Int. J. Nonlinear Sciences and Numerical Simulation. 2 (2005), 163-168.

[9] Erdem Cuce, Pinar Mert Cuce, A successful application of homotopy perturbation method for efficiency and effectiveness assessment of longitudinal porous fins, Energy Conversion and Management, 93 (2015), 92-99.

[10] Ghasemi M, Tavassoli Kajani M., Babolian E, Application of Hes homotopy perturbation method to nonlinear integro-differential equations, Applied Mathematics and Computation, 188 (2007), 538-548.

[11] He J.H, Homotopy perturbation technique, Computer Methods in Applied Mechanics and Engineering, 178 (1999), 257-262.

[12] He J.H, A coupling method of homotopy technique and perturbation technique for nonlinear problems, International Journal of Non-Linear Mechanics, 35 (2000), 37-43.

[13] He J.H, Homotopy perturbation method: a new nonlinear analytical technique, Applied Mathematics and Computation, 135 (2003), 73-79.

[14] He J.H, Comparison of homotopy perturbation method and homotopy analysis method, Applied Mathematics and Computation, 156 (2004), 527-539.

[15] Khader M.M., Ahmed M. M., Numerical simulation using the finite difference method for the flow and heat transfer in a thin liquid film over an unsteady stretching sheet in a saturated porous medium in the presence of thermal radiation. J King Saud University, 25 (2013), 29-34. 
[16] Laraqi N., Rashidi M.M., Garcia de Maria J.M., Baïri A., Analytical model for the thermo-hydrodynamic behaviour of a thin lubricant film, Tribology International, 40 (2011), 1083-1086.

[17] Makinde O.D., Aziz A., Boundary layer flow of a nanofluid past a stretching sheet with a convective boundary condition, Int. J. of Thermal Sciences, 50 (2011), 1326-1332.

[18] Masoud S., Martin H., Hadi E. K., The comparison of homotopy perturbation method with finite difference method for determination of maximum beam deflection, $J$. of Theoretical and Applied Physics, 7 (2013), 1-8.

[19] Noor M.A., Mohyud-Din S.T., An efficient algorithm for solving fifth order boundary value problems, Mathematical and computer modelling, 45 (2007), 954-964.

[20] Noor N.F.M, Hashim I, Thermocapillarity and magnetic field effects in a thin liquid film on an unsteady stretching surface, it Int. J. of Heat and Mass Transfer, 53 (2010), 2044-2051.

[21] Susanta M., Thermocapillary flow of thin liquid film over a porous stretching sheet in presence of suction/injection, Int. J. of Heat and Mass Transfer, 70 (2014), 819-826

[22] Usha R, Sridharan R, On the motion of a liquid film on an unsteady stretching surface, $J$. of Fluids Engineering, 117 (1995), 81-85.

[23] Wang C, Pop I, Analysis of the flow of a power-law fluid film on an unsteady stretching surface by means of homotopy analysis method, J. of Non-Newtonian Fluid Mechanics, 138 (2006), 161-172.

[24] Wang C.Y, Stretching surface of a rotating fluid, Zeitschrift für angewandte Mathematik und Physik ZAMP, 39 (1988), 177-185.

[25] Wang C.Y, Liquid film on an unsteady stretching surface, Wang C.Y, 1990. Liquid film on an unsteady stretching surface. Quart Appl. Math. 48, 601-610. , 48 (1990), 601-610. 